\title{
DIAGNÓSTICO GENERAL DE LA SITUACIÓN DE VIOLENCIA Y CRIMINALIDAD EN CARTAGENA DE INDIAS. 2008 - 2013
}

\author{
Fredi E Goyeneche González¹, Jerry Pardo Gómez², Oswaldo Mármol Pérez ${ }^{3}$
}

\begin{abstract}
RESUMEN
El Centro de Observación y Seguimiento del Delito, COSED, es la principal fuente de información y acopio estadístico de las principales expresiones de la violencia y la criminalidad en la ciudad de Cartagena, y principal insumo de apoyo a las administraciones distritales en la formulación de estrategias de prevención y mitigación del fenómeno delictivo en la ciudad. El presente documento toma como fuente principal al COSED y muestra un análisis descriptivo que sirve como diagnóstico preliminar de las principales expresiones de la violencia en la ciudad de Cartagena. Se utiliza una metodología de corte descriptivo en la que se analizan 7 diferentes variables delictivas englobadas en 3 grandes dimensiones que definen la incidencia delictiva en la ciudad: Delitos contra la vida, contra la salud y contra la propiedad privada y el patrimonio.
\end{abstract}

Los principales resultados datan de un aumento sostenido en el número de homicidios, los cuales a su vez presentan una coincidencia espacial con aquellos barrios que muestran los peores indicadores sociales como pobreza, empleo, salud, educación, entre otros. Otras expresiones delictivas como la violencia intrafamiliar, los delitos sexuales y las extorsiones también muestran esta coincidencia y solo los hurtos parecen distribuirse mayormente en barrios y comunas de estratificación socioeconómica alta.

\section{PALABRAS CLAVES}

Incidencia delictiva, desarrollo social, expresiones de la violencia, pobreza

\begin{abstract}
The center of Observation and Monitoring of the Crime, COSED, is the main source of information and statistical collection of the principal expressions of violence and criminality in Cartagena, and it is also the main source of support for the district administrations in the creation of prevention strategies and mitigation of the criminal phenomenon in the city. This document is based on COSEC as its main source and it shows a descriptive analysis that serves as a preliminary diagnosis of the main expressions of violence in Cartagena. A descriptive methodology is used, with which we analyze 7 different criminal variables grouped in 3 large dimensions that define the criminal incidence in the city: crimes against life, health, private property and the patrimony.
\end{abstract}

The main results come from a steady increase in the number of homicides, which present a location coincidence with those neighborhoods that show the worst social indicators such as poverty, employment, health, and education among others. Other criminal expressions such as domestic violence, sexual crimes, and extortion also show this coincidence. Only thefts seem to distribute mainly in neighborhoods and communes with a high economic stratification.

\section{KEYWORDS}

Crime Incidence, Social Development, Expressions of Violence, Poverty.

Depositado en agosto 15 de 2014, aprobado en octubre 31 de 2014..

Este artículo hace parte de los resultados del Grupo de Investigación Violencia y Ciudad.

1 Economista. Phd (c) en Ciencias Sociales. Msc. En Desarrollo Social. Docente universitario. Director del Centro de Observación y Seguimiento del Delito, COSED. Líder Grupo de Investigación Economía, Violencia y Ciudad. fredigoyeneche@gmail.com 2 Economista. Msc. En Desarrollo Sostenible y Ambiente. Investigador del Centro de Observación y Seguimiento del Delito, COSED. Integrante Grupo de Investigación Economía, Violencia y Ciudad. Jerrywise70@yahoo.es

3 Economista. Investigador del Centro de Observación y Seguimiento del Delito, COSED. Integrante Grupo de Investigación Economía, Violencia y Ciudad. oswmarmol@hotmail.com 


\section{INTRODUCCIÓN}

El análisis de la información producto de la sistematización de las fuentes oficiales resulta ser, sin dudas, preocupante cuando se habla de violencia y criminalidad. El aumento de los homicidios, por ejemplo, durante el año 2013 estuvo en el orden de 48 casos más que en el año anterior. Las cifras son 256 y 264 para los años 2012 y 2013 respectivamente, es decir, un aumento porcentual del $3,1 \%$.

Las características de los homicidios que se presentan en el presente documento permiten observar los elementos caracterizantes que son comunes y que dan una idea del perfil del fenómeno social. Este perfil muestra que las víctimas de homicidios y los barrios donde estos mayormente ocurren son barrios pobres en los que prevalece el estrato 1. Por otra parte, el perfil laboral infiere la condición de ser población vinculada a la economía informal. Son individuos varones y jóvenes.

Las cifras se pueden interpretar como surgidas de dos razones principales para iniciar la comprensión de la dinámica homicida en la ciudad. Por una parte, homicidios en la modalidad de sicariatos involucra criterios económicos, manejo gerencial e igualmente una racionalidad de eficiencia. Serían los homicidios que responden a razones de carácter económico.

Pero también podríamos interpretar las cifras desde la perspectiva de la Violencia Intrafamiliar, riñas entre pandillas o riñas personales como expresión de una baja valoración de la tolerancia social. En ese contexto, llama la atención sobre la dinámica que están adquiriendo los homicidios en un entramado social que se percibe con baja credibilidad institucional en el cartagenero de la calle.

\section{REVISIÓN DE LA LITERATURA}

La siguiente revisión sirve de base para conocer algunos referentes literarios que han servido de apoyo para argumentar los análisis aquí mostrados. Se refieren a documentos del orden local, nacional e internacional, y básicamente son extraídos de bases de datos bibliográficas de reconocimiento, así como de revistas científicas de alto impacto.

En primer lugar cabe referenciar a Llorente, Escobedo \& Rubio (2002) con "Violencia homicida y estructuras criminales en Bogotá", el cual posee mucha similitud con el presente documento en tanto a que muestra un marco evolutivo del comportamiento del homicidio en términos diagnósticos y utilizando también una metodología descriptiva. En él se encontró que se concluyó que en Bogotá predomina la violencia instrumental antes que la impulsiva, que los homicidios están concentrados en pocos focos críticos, los cuales son persistentes en el tiempo y se pueden asimilar a un escenario de difusión contagiosa de los homicidios.

Sobresale también Sarmiento \& Becerra (1998) con un documento denominado Análisis de las relaciones entre violencia y equidad, en el que se construyó una base de datos de los municipios de todo el país y se utilizó como herramienta cuantitativa, una regresión multivariante, de corte transversal para el año 1993. En él se concluye que la violencia tiende a reproducirse con más probabilidad en sectores o zonas con altas posibilidades de acumulación económica y con presencia débil del Estado. Sin embargo, en las zonas y grupos de población con niveles altos de violencia, los pobres son principalmente las víctimas. Ello ha servido de base para la formulación de estrategias de mitigación y atención del fenómeno de la violencia en las ciudades, lo cual es similar a las pretensiones que se tienen con el desarrollo de este documento

Uno de los trabajos más destacados de las últimas décadas en el país fue desarrollado por Rubio (1997) y se titula Costos de la violencia en Colombia. Como objetivo principal, el autor se plantea ofrecer algunos elementos para enriquecer el debate sobre las prioridades de acción pública en materia de prevención y control de la violencia. La metodología utilizada es la de un completo análisis estadístico de las cifras de violencia en Colombia y a través de una profunda revisión de la literatura que evidencia el fenómeno en cuestión. Una de las 
conclusiones más contundentes de este ensayo, es que las políticas públicas deben estar basadas, ante todo, en un conocimiento razonable de lo que está pasando en base a dos pilares: los datos sobre la realidad y alguna teoría para detectar las interrelaciones entre estos.

En el ámbito local sobresale el trabajo de Goyeneche, Pardo, \& Mármol (2011), Valoración Económica del delito de homicidio en Cartagena. 1995 - 2005, en el que se plantea como principal objetivo estimar el valor monetario de la vida de una persona que ha sido víctima de un homicidio, usando la metodología Años Potenciales de Vida Perdidos (APVP). EN este documento se realiza en completo diagnóstico de la situación de la violencia homicida para el periodo de estudio, lo cual se convierte en fundamento básico para tener en cuenta en desarrollo del presente documento, ya que la metodología descriptiva que fue usada, es muy similar a la que aquí se propone

Por último, resaltar el trabajo de Becker (1968), El Crimen y el Castigo: Un Enfoque Económico, en el que se describe un modelo que consiste en aplicar a todo tipo de actividades humanas la metodología de la teoría económica convencional. En concreto establece que un criminal es un agente maximizador. En este sentido, afirma que, ceteris paribus, al aumentar la probabilidad de condena disminuye el número de crímenes cometidos por un individuo. Llego a la conclusión de que las decisiones óptimas son interpretadas como aquellas en las que finalmente se minimiza la perdida social en relación a los ingresos de los crímenes.

\section{METODOLOGÍA}

\section{TIPO DE ESTUDIO}

Dada la naturaleza de este estudio, la cual se basa principalmente en la intención de extraer aspectos característicos que permitan la identificación de factores del entorno, que configuran la dinámica de las expresiones de la violencia y criminalidad en la ciudad de Cartagena de Indias. La tipología en la que se enmarca es la de tipo "Descriptivo" (Hurtado, 2010).

\section{PROCEDIMIENTO}

Inicialmente se tienen en cuenta las expresiones de la violencia que han sido consideradas por la Secretaría del Interior en construcción del Plan Integral para la Seguridad y Convivencia Ciudadana de Cartagena, distribuidas en 3 grandes dimensiones de la siguiente manera:

Ilustración No 1. Dimensiones y categorías de las expresiones delictivas en la ciudad de Cartagena
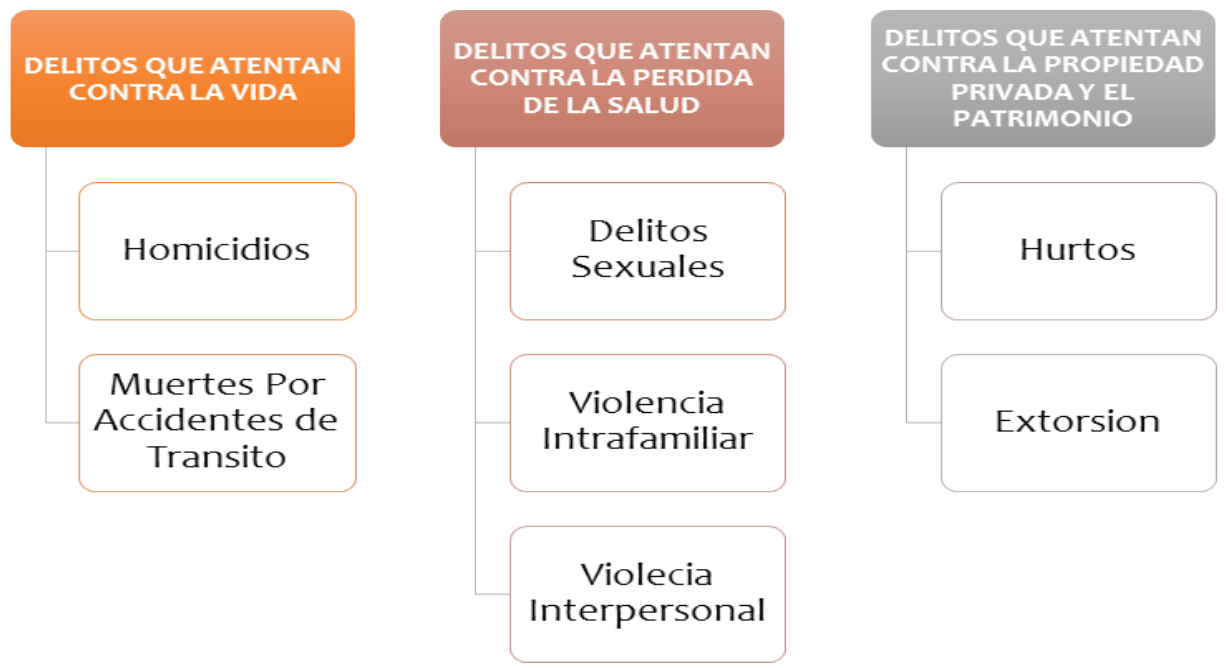

Fuente. Autores con base en COSED y Secretaría del Interior 
El lector notará que cada dimensión incluye dos o más expresiones delictivas. Véase que la dimensión que describe los Delitos que Atentan contra la Vida, incluye a los homicidios y las Muertes en Accidentes de Tránsito. Asimismo los delitos relacionados con la pérdida de la salud se describen a través de la incidencia de la violencia sexual, la violencia interpersonal y la violencia intrafamiliar. Por último, la categoría correspondiente a los delitos contra la propiedad privada y el patrimonio hace referencia a los delitos de hurtos y extorsión.

Posteriormente, se efectúa una recopilación de información necesaria para la descripción de las principales variables que caracterizan la violencia y la criminalidad en la ciudad de Cartagena a través de la construcción de una base de datos que será procesada a través de la utilización de los Softwares Microsoft Excel y SPSS.

El análisis descriptivo se hará utilizando las herramientas básicas de la estadística como las gráficas de barras y tortas, tablas de frecuencia, entre otros, adicionando un enfoque espacial que incluye la georreferenciación de cada expresión delictiva a través del diseño de mapas.

\section{TIPO DE FUENTE}

Se hace uso de bases de datos e información procedente de fuentes oficiales encargadas de la recopilación y sistematización de este tipo de variables, como el COSED, Instituto Nacional de Medicina Legal y Ciencias Forenses, DATT, CTI, entre otros. Por tanto, se clasifican como fuentes de tipo Secundaria

\section{RESULTADOS Y DISCUSIÓN}

En orden de vislumbrar los principales aspectos característicos de las expresiones de la violencia tenidas en cuenta en desarrollo del presente documento, se presentan los resultados del mismo.

\section{DELITOS QUE ATENTAN CONTRA LA VIDA}

\section{HOMICIDIOS}

\section{Gráfica No 1. Homicidios 2008 - 2013}

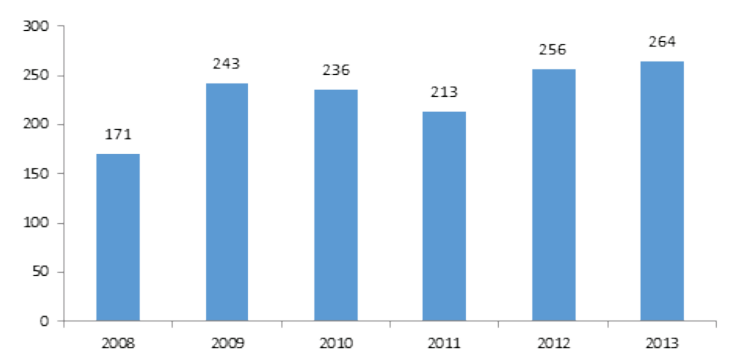

Fuente. Autores con base en COSED y Secretaría del Interior

Los Homicidios en Cartagena vienen registrando incrementos desde el año 2012, cuando el registro total anual fue de 256 casos, después de haber registrado en el 2011, 213 en total, es decir, que el incremento porcentual de los homicidios en el años 2012 fue del 20\%, mientras que en el año 2013, los homicidios sumaron 264 muertes, luego de que en el 2012 fueran 256, lo que representa un incremento del $3 \%$. De esta forma se evidencia que los homicidios en Cartagena durante los últimos 2 años han venido incrementando, pero dicho incremento es $17 \%$ menor en el 2013 al registrado en el 2012.

En el comportamiento general durante el periodo 2008 - 2013, la tendencia de los homicidios es claramente ascendente, al pasar de 171 casos en el 2008 a 264 en el 2013 lo que indica un incremento del $54 \%$, de manera que el número de homicidios registrados en la actualidad crecieron en más de la mitad del registro presentado hace 6 años. El año 2013 presentó la mayor cifra de homicidios de los últimos 6 años y durante todo el periodo 2008 - 2013. Solo en dos años hubo decrecimiento en el número de homicidios (años 2010 y 2011) ver gráfica 1.

Las características de las víctimas de homicidios, siguen siendo similares a las observadas en otros años; en su mayoría, las víctimas son de sexo masculino y jóvenes entre los 20 y 39 años de edad. Sin embargo, hay que destacar que durante el año 2013 hubo importante registro de víctimas entre los 10 y los 19 años de edad, 44 en total, cifra no registrada en ninguno de los 5 años inmediatamente anteriores y con un gran componente de víctimas menores de edad. 
Tabla No 1. Homicidios 2008 - 2013 según edad y sexo de la víctima

\begin{tabular}{|c|c|c|c|c|c|c|c|c|c|c|c|c|c|}
\hline \multirow{2}{*}{ Edad } & \multicolumn{2}{|c|}{2008} & \multicolumn{2}{|c|}{2009} & \multicolumn{2}{|c|}{2010} & \multicolumn{2}{|c|}{2011} & \multicolumn{3}{|c|}{2012} & \multicolumn{2}{|c|}{2013} \\
\hline & $\mathrm{F}$ & M & $\mathrm{F}$ & M & $\mathrm{F}$ & M & $\mathrm{F}$ & M & $\mathrm{F}$ & M & $\mathrm{N} / \mathrm{E}$ & $\mathrm{F}$ & M \\
\hline De $0-4$ & 0 & 0 & 1 & 0 & 0 & 1 & 0 & 1 & 0 & 0 & 0 & 0 & 0 \\
\hline De 5 - 9 & 0 & 0 & 0 & 0 & 1 & 0 & 0 & 1 & 0 & 0 & 0 & 0 & 0 \\
\hline De 10 - 14 & 0 & 0 & 1 & 0 & 0 & 1 & 0 & 1 & 0 & 2 & 0 & 1 & 2 \\
\hline De $15-19$ & 1 & 23 & 1 & 19 & 1 & 23 & 6 & 28 & 2 & 35 & 0 & 2 & 41 \\
\hline De 20 - 24 & 1 & 21 & 1 & 44 & 1 & 32 & 3 & 36 & 1 & 63 & 0 & 5 & 62 \\
\hline De $25-29$ & 2 & 30 & 1 & 34 & 2 & 35 & 2 & 30 & 8 & 37 & 0 & 0 & 45 \\
\hline De 30 - 34 & 1 & 24 & 1 & 41 & 1 & 32 & 3 & 22 & 2 & 31 & 0 & 2 & 39 \\
\hline De 35 - 39 & 2 & 24 & 1 & 38 & 1 & 36 & 2 & 22 & 1 & 27 & 0 & 2 & 17 \\
\hline De $40-44$ & 5 & 14 & 1 & 26 & 2 & 27 & 2 & 17 & 0 & 17 & 0 & 0 & 18 \\
\hline De 45 - 49 & 1 & 9 & 1 & 16 & 2 & 17 & 0 & 16 & 0 & 13 & 0 & 2 & 9 \\
\hline De $50-54$ & 0 & 4 & 0 & 6 & 1 & 5 & 3 & 7 & 1 & 4 & 0 & 1 & 6 \\
\hline De $55-59$ & 0 & 2 & 0 & 6 & 1 & 7 & 1 & 3 & 0 & 5 & 0 & 2 & 1 \\
\hline De $60-64$ & 0 & 2 & 0 & 2 & 1 & 2 & 0 & 1 & 0 & 0 & 0 & 0 & 2 \\
\hline De $65-69$ & 0 & 3 & 0 & 0 & 0 & 2 & 0 & 3 & 0 & 4 & 0 & 0 & 1 \\
\hline 70 o más & 0 & 2 & 0 & 0 & 0 & 1 & 1 & 1 & 0 & 2 & 0 & 0 & 2 \\
\hline $\mathrm{S} / \mathrm{D}$ & 0 & 0 & 0 & 2 & 0 & 1 & 0 & 1 & 0 & 0 & 1 & 0 & 2 \\
\hline Total general & 13 & 158 & 9 & 234 & 14 & 222 & 23 & 190 & 15 & 240 & 1 & 17 & 247 \\
\hline
\end{tabular}

Fuente. Autores con base en COSED y Secretaría del Interior

Tabla No 2. Homicidios 2008 - 2013 por modalidad

\begin{tabular}{|c|c|c|c|c|c|c|c|}
\hline Modalidad & 2008 & 2009 & 2010 & 2011 & 2012 & 2013 & Total general \\
\hline Sicariato & 78 & 149 & 116 & 92 & 97 & 102 & 634 \\
\hline Riña común & 38 & 40 & 70 & 57 & 70 & 60 & 335 \\
\hline Hurto & 20 & 13 & 10 & 11 & 18 & 32 & 104 \\
\hline Riña entre pandillas & 0 & 0 & 0 & 0 & 37 & 44 & 81 \\
\hline Desconocida o por establecer & 9 & 16 & 7 & 13 & 16 & 14 & 75 \\
\hline Bala perdida & 2 & 7 & 11 & 8 & 3 & 2 & 33 \\
\hline Ajuste de cuentas & 4 & 6 & 13 & 6 & 1 & 0 & 30 \\
\hline Violencia de género & 13 & 2 & 2 & 8 & 2 & 3 & 30 \\
\hline Violencia intrafamiliar & 0 & 4 & 2 & 2 & 7 & 5 & 20 \\
\hline Ciudadano en defensa propia & 5 & 1 & 2 & 8 & 2 & 0 & 18 \\
\hline Accidental & 0 & 0 & 0 & 6 & 3 & 1 & 10 \\
\hline Otro tipo & 2 & 2 & 1 & 2 & 0 & 0 & 7 \\
\hline Intolerancia & 0 & 1 & 2 & 0 & 0 & 0 & 3 \\
\hline Linchamiento & 0 & 2 & 0 & 0 & 0 & 0 & 2 \\
\hline Extorsión & 0 & 0 & 0 & 0 & 0 & 1 & 1 \\
\hline Total general & 171 & 243 & 236 & 213 & 256 & 264 & 1383 \\
\hline
\end{tabular}

Fuente. Autores con base en COSED y Secretaría del Interior 
La modalidad preponderante sigue siendo el Sicarito, que para el 2013 aumentó de 97 a 102 casos, que desde el año 2011 mantiene una tendencia al alza y que durante todo el periodo 2008 - 2013 ha representado en promedio el $46 \%$ del total de homicidios ocurridos. Por su parte, los homicidios ocurridos por riñas, tienen la segunda mayor representatividad en el periodo concentrando el $30 \%$ del total de homicidios y sumando en el año 2013, 104, entre riñas comunes y riñas de pandillas, 3 menos que las ocurridas en 2012, pero como se puede observar en la tabla, mientras la riñas comunes disminuyeron, 10 casos, las riñas de pandillas aumentaron en 7 .

Tabla No 3. Homicidios 2008 - 2013 según 20 primeros barrios de ocurrencia

\begin{tabular}{|c|c|c|c|c|c|c|c|}
\hline Barrio & 2008 & 2009 & 2010 & 2011 & 2012 & 2013 & Total general \\
\hline Olaya Herrera(*) & 23 & 28 & 12 & 23 & 36 & 28 & 150 \\
\hline El Pozón & 13 & 20 & 13 & 15 & 22 & 18 & 101 \\
\hline Nelson Mandela & 1 & 8 & 9 & 8 & 9 & 15 & 50 \\
\hline La Esperanza & 7 & 4 & 8 & 3 & 8 & 12 & 42 \\
\hline La Candelaria & 3 & 5 & 8 & 3 & 10 & 9 & 38 \\
\hline La María & 1 & 4 & 7 & 3 & 8 & 10 & 33 \\
\hline Barrio Chino & 6 & 9 & 7 & 4 & 3 & 3 & 32 \\
\hline San José de los Campanos & 1 & 2 & 4 & 5 & 6 & 10 & 28 \\
\hline Fredonia & 5 & 5 & 5 & 0 & 8 & 5 & 28 \\
\hline Torices & 2 & 7 & 6 & 2 & 5 & 5 & 27 \\
\hline San Pedro Mártir & 1 & 3 & 9 & 5 & 4 & 3 & 25 \\
\hline San Francisco & 3 & 4 & 3 & 5 & 4 & 5 & 24 \\
\hline Nuevo Paraíso & 3 & 3 & 1 & 4 & 4 & 9 & 24 \\
\hline San Fernando & 0 & 5 & 5 & 2 & 3 & 9 & 24 \\
\hline Ternera & 3 & 6 & 7 & 1 & 4 & 2 & 23 \\
\hline Boston & 2 & 2 & 1 & 4 & 6 & 7 & 22 \\
\hline Republica del Líbano & 3 & 5 & 4 & 1 & 5 & 3 & 21 \\
\hline El Bosque & 4 & 6 & 2 & 4 & 2 & 2 & 20 \\
\hline Zaragocilla & 3 & 4 & 1 & 5 & 2 & 1 & 16 \\
\hline Henequén & 1 & 3 & 2 & 6 & 3 & 0 & 15 \\
\hline
\end{tabular}

Fuente. Autores con base en COSED y Secretaría del Interior

El análisis de los homicidios por barrio de ocurrencia permite señalar ciertas particularidades; lo primero, es el considerable aumento en los barrios San Fernando, San José de Los Campanos, Nelson Mandela y La Esperanza; lo segundo, es la observable disminución en los barrios Olaya Herrera y el Pozón, los cuales para el año 2012 mostraron aumentos en el número de casos con respecto al año inmediatamente anterior. Ciertamente, la interpretación resulta ser ambigua ya que si bien es resaltable la labor de las autoridades y demás entes pertinentes en la reducción de la criminalidad en los barrios que históricamente marcaban cifras altas, el esfuerzo no alcanza a ser suficiente para cubrir aquellos barrios en los que las cifras no eran tan preponderantes. Por tal motivo, el lector debe entender que los resultados observados no pueden ser atribuidos únicamente a la labor de las autoridades, sino que es necesaria la existencia de un apoyo y colaboración de la ciudadanía para con éstas. Por otro lado, las cifras según zonas siguen mostrando un perfil netamente urbano con 257 casos. 


\section{Ilustración No 2. Distribución geográfica de los homicidios en Cartagena de Indias durante el 2013, según densidad de Kernel}

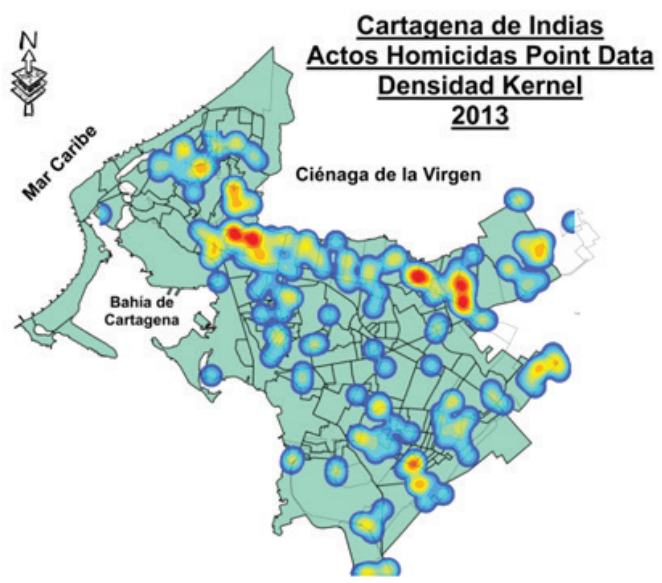

Fuente. Cosed

La distribución geográfica de Kernel comprueba que los homicidios están concentrados en la zona más marginal de la ciudad de Cartagena, y son, entonces, la Localidad de la Virgen y Turística y sus Unidades Comuneras 4, 5 y 6 los territorios que mayor número de homicidios concentran, considerándose los puntos de fuego de la violencia homicida de la ciudad de Cartagena.

\section{MUERTES EN ACCIDENTES DE TRÁNSITO}

Gráfica No 2. Muertes en accidente de tránsito $2008-2013$

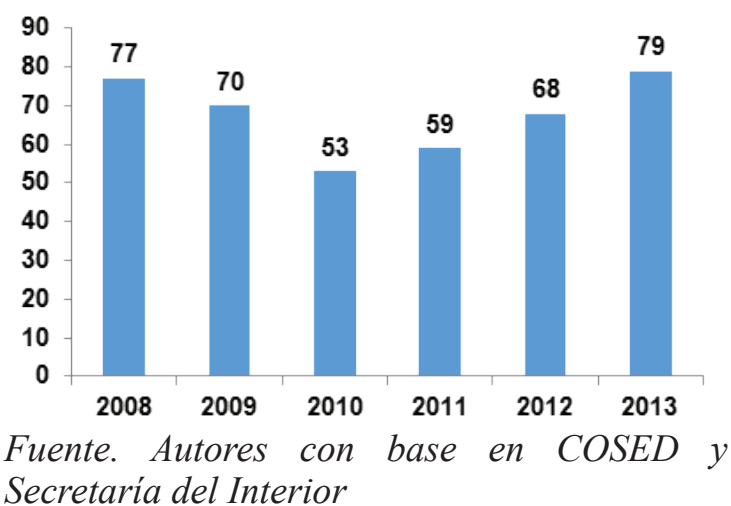

El comportamiento de las muertes por accidentes de tránsito en Cartagena durante el periodo 2008
- 2013 permite constatar que desde el año 2011 el total de este tipo de muertes ha superado en número al del año inmediatamente anterior, lo que deja una tendencia creciente que consolida el año 2013, como el año con mayor cantidad de muertes en accidente de tránsitos de los 6 años.

Las principales características de las víctimas, se observa en la distribución de casos según edades y sexo. Durante el año 2013 hubo un total de 67 víctimas de sexo masculino y las 12 restantes del sexo contrario. En este este año también se registró el mayor número de muertes en personas de sexo masculino con edades de entre 25 y 29 años de los últimos 6 años, así como la mayor cantidad de adultos mayores de 70 años pertenecientes al mismo sexo.

Los Peatones atropellados, a diferencia de lo observado durante el año 2012, fue el tipo de accidente más frecuente en el 2013, después que fuera el más común la colisión entre objetos móviles. En general, las cifras reflejan un aumento del $32 \%$ en el total de atropellamientos entre 2012 y 2013 y una reducción cercana al $28 \%$ en el número de colisiones móviles.

La territorialización de las muertes en accidente de tránsito en la ciudad de Cartagena permite destacar al barrio Olaya Herrera como el de mayor número de casos, como también ocurre en las muertes por homicidios, pero esta cifra es inferior en el 2013 a la registrada en el 2012, en un solo caso. Por su parte, en todo el periodo analizado los barrios Mamonal, Bosque, El Pozón, Bayunca y Ceballos, después de Olaya, tienen la mayor frecuencia, entre más de 200 barrios en los que hay evidencia de casos en la ciudad tanto en su zona urbana como rural.

\section{DELITOS QUE ATENTAN CONTRA LA PÉRDIDA DE LA SALUD}

\section{DELITOS SEXUALES}

Los delitos sexuales denunciados en la ciudad de Cartagena durante el periodo 2008 - 2013 tienen una clara tendencia creciente desde el año 2010 , cuando se obtuvo la menor cantidad de denuncias por este delito, 339 en total, pero alcanzaron el mayor registro del periodo en el 
DIAGNÓSTICO GENERAL DE LA SITUACIÓN DE VIOLENCIA Y

CRIMINALIDAD EN CARTAGENA DE INDIAS. 2008 - 2013

Tabla No 4. Muertes en accidentes de tránsito según edad y sexo 2008 - 2013

\begin{tabular}{|c|c|c|c|c|c|c|c|c|c|c|c|c|}
\hline \multirow{2}{*}{ Rango Edad } & \multicolumn{2}{|c|}{2008} & \multicolumn{2}{|c|}{2009} & \multicolumn{2}{|c|}{2010} & \multicolumn{2}{|c|}{2011} & \multicolumn{2}{|c|}{2012} & \multicolumn{2}{|c|}{2013} \\
\cline { 2 - 14 } & F & M & F & M & F & M & F & M & F & M & F & M \\
\hline De $0-4$ & 0 & 0 & 1 & 0 & 1 & 0 & 0 & 1 & 0 & 0 & 1 & 1 \\
\hline De $5-9$ & 0 & 0 & 0 & 0 & 0 & 1 & 0 & 1 & 1 & 2 & 0 & 0 \\
\hline De $10-14$ & 0 & 0 & 1 & 1 & 1 & 0 & 0 & 0 & 0 & 1 & 0 & 1 \\
\hline De $15-19$ & 0 & 6 & 0 & 4 & 0 & 0 & 0 & 4 & 2 & 2 & 2 & 7 \\
\hline De $20-24$ & 0 & 6 & 1 & 9 & 0 & 0 & 2 & 7 & 0 & 9 & 0 & 6 \\
\hline De $25-29$ & 0 & 11 & 1 & 8 & 0 & 4 & 0 & 6 & 2 & 7 & 1 & 12 \\
\hline De $30-34$ & 0 & 13 & 1 & 5 & 2 & 6 & 0 & 4 & 1 & 4 & 0 & 8 \\
\hline De $35-39$ & 1 & 6 & 1 & 3 & 0 & 6 & 0 & 4 & 1 & 3 & 2 & 4 \\
\hline De $40-44$ & 0 & 4 & 1 & 7 & 1 & 6 & 1 & 2 & 1 & 4 & 1 & 3 \\
\hline De $45-49$ & 1 & 5 & 0 & 8 & 1 & 3 & 2 & 2 & 0 & 2 & 3 & 2 \\
\hline De 50 - 54 & 2 & 3 & 0 & 1 & 0 & 2 & 0 & 6 & 1 & 8 & 0 & 2 \\
\hline De 55 - 59 & 1 & 1 & 0 & 3 & 0 & 4 & 0 & 3 & 4 & 7 & 0 & 2 \\
\hline De 60 - 64 & 0 & 3 & 1 & 2 & 1 & 3 & 1 & 2 & 0 & 0 & 0 & 5 \\
\hline De 65 - 69 & 1 & 1 & 0 & 3 & 0 & 3 & 1 & 2 & 0 & 1 & 0 & 4 \\
\hline 70 o Más & 2 & 10 & 3 & 5 & 0 & 6 & 2 & 4 & 1 & 4 & 2 & 10 \\
\hline SIN DATO & 0 & 0 & 0 & 0 & 1 & 1 & 0 & 2 & 0 & 0 & 0 & 0 \\
\hline Total & 8 & 69 & 11 & 59 & 8 & 45 & 9 & 50 & 14 & 54 & 12 & 67 \\
\hline
\end{tabular}

Fuente. Autores con base en COSED y Secretaría del Interior

Tabla No 5. Muertes en accidente de tránsito 2008 - 2013 según clase de accidente

\begin{tabular}{|c|c|c|c|c|c|c|c|}
\hline Clase de accidente & 2008 & 2009 & 2010 & 2011 & 2012 & 2013 & Total general \\
\hline Peatón atropellado & 25 & 19 & 24 & 24 & 22 & 30 & 144 \\
\hline Colisión con objeto móvil & 27 & 27 & 18 & 21 & 28 & 25 & 146 \\
\hline Colisión con objeto fijo & 10 & 12 & 4 & 4 & 7 & 11 & 48 \\
\hline Caída de ocupante & 2 & 3 & 2 & 4 & 4 & 5 & 20 \\
\hline Volcamiento & 5 & 4 & 0 & 2 & 4 & 3 & 18 \\
\hline Ciclista atropellado & 3 & 3 & 3 & 2 & 0 & 0 & 11 \\
\hline Sin dato & 2 & 0 & 1 & 1 & 1 & 5 & 10 \\
\hline Otra clase de accidente & 3 & 1 & 1 & 1 & 2 & 0 & 8 \\
\hline Colisión con animales & 0 & 1 & 0 & 0 & 0 & 0 & 1 \\
\hline Total general & 77 & 70 & 53 & 59 & 68 & 79 & 406 \\
\hline
\end{tabular}

Fuente. Autores con base en COSED y Secretaría del Interior 
Tabla No 6. Muertes en accidente de tránsito 2008 - 2013 según 20 primeros barrios de ocurrencia

\begin{tabular}{|c|c|c|c|c|c|c|c|}
\hline Barrio & 2008 & 2009 & 2010 & 2011 & 2012 & 2013 & Total general \\
\hline Olaya Herrera $\left(^{*}\right)$ & 3 & 4 & 2 & 1 & 6 & 5 & 21 \\
\hline Mamonal & 5 & 1 & 2 & 1 & 4 & 7 & 20 \\
\hline Bosque & 7 & 3 & 1 & 5 & 0 & 0 & 16 \\
\hline El Pozón & 2 & 3 & 3 & 4 & 0 & 0 & 12 \\
\hline Bayunca & 0 & 0 & 0 & 2 & 4 & 5 & 11 \\
\hline Ceballos & 2 & 1 & 2 & 0 & 4 & 2 & 11 \\
\hline Blas de Lezo & 1 & 0 & 2 & 5 & 1 & 0 & 9 \\
\hline San Isidro & 0 & 3 & 2 & 1 & 0 & 3 & 9 \\
\hline Torices & 2 & 1 & 1 & 1 & 1 & 3 & 9 \\
\hline El Bosque & 1 & 0 & 0 & 0 & 4 & 4 & 9 \\
\hline La Esperanza & 1 & 4 & 2 & 0 & 1 & 0 & 8 \\
\hline Martínez Martelo & 2 & 1 & 1 & 0 & 1 & 3 & 8 \\
\hline La Boquilla & 3 & 4 & 0 & 0 & 0 & 1 & 8 \\
\hline La Quinta & 1 & 2 & 2 & 1 & 0 & 1 & 7 \\
\hline San José de los Campanos & 1 & 0 & 1 & 1 & 2 & 2 & 7 \\
\hline Alcibia & 0 & 4 & 1 & 1 & 1 & 0 & 7 \\
\hline La María & 2 & 3 & 0 & 2 & 0 & 0 & 7 \\
\hline Barrio Chino & 0 & 0 & 3 & 0 & 2 & 1 & 6 \\
\hline Sin dato & 0 & 1 & 0 & 3 & 0 & 2 & 6 \\
\hline Las Gaviotas & 0 & 1 & 2 & 1 & 0 & 2 & 6 \\
\hline
\end{tabular}

Fuente. Autores con base en COSED y Secretaría del Interior

Gráfica No 3. Delitos sexuales 2008 - 2013

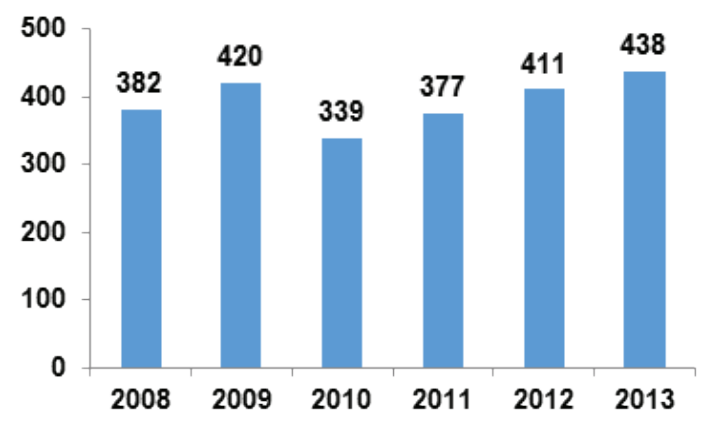

Fuente. COSED con base en Medicina Legal

2013, 438 casos. Aunque la cifra del 2010 marca un decrecimiento importante con respecto a los años inmediatamente anteriores, el incremento en la cifras en todo del periodo 2008 - 2013 es contundente, el $15 \%$, debido a que es este año el único inferior a su año inmediatamente anterior.
Las víctimas de los delitos sexuales en Cartagena en su gran mayoría son de sexo femenino y con edades entre los 0 y 14 años, mayoritariamente, en promedio solo un $16 \%$ del total de casos ocurridos fueron contra personas de sexo masculino y cerca de un $12 \%$ contra personas mayores de 19 años.

Los barrios con mayor frecuencia de casos de delitos sexuales, así como en los demás delitos de impacto en la ciudad de Cartagena, son Olaya Herrera, El Pozón y Nelson Mandela y aparece en cuarto lugar el barrio San José de los Campanos, que también está presente entre los 20 más comunes en todos los delitos. Estos barrio como ya se ha dicho, pertenecen a los estratos promedios 1 y 2 y tienen las perores condiciones de vida digna en la ciudad. 
DIAGNÓSTICO GENERAL DE LA SITUACIÓN DE VIOLENCIA Y CRIMINALIDAD EN CARTAGENA DE INDIAS. 2008 - 2013

Tabla No 7. Delitos sexuales 2008 - 2013 por edad y sexo de la víctima

\begin{tabular}{|c|c|c|c|c|c|c|c|c|c|c|c|c|}
\hline \multirow{2}{*}{ Edad } & 2008 & & 2009 & & 2010 & & 2011 & & 2012 & & 2013 & \\
\hline & $\mathrm{F}$ & $\mathrm{M}$ & $\mathrm{F}$ & $\mathrm{M}$ & $\mathrm{F}$ & $\mathrm{M}$ & $\mathrm{F}$ & $\mathrm{M}$ & $\mathrm{F}$ & $\mathrm{M}$ & $\mathrm{F}$ & $\mathrm{M}$ \\
\hline De $0-4$ & 38 & 9 & 59 & 17 & 41 & 11 & 38 & 7 & 34 & 15 & 38 & 15 \\
\hline De $5-9$ & 69 & 34 & 82 & 40 & 63 & 19 & 70 & 25 & 67 & 23 & 76 & 21 \\
\hline De $10-14$ & 110 & 13 & 120 & 9 & 95 & 8 & 114 & 10 & 144 & 18 & 161 & 16 \\
\hline De $15-19$ & 56 & 2 & 45 & 5 & 55 & 3 & 45 & 11 & 56 & 9 & 50 & 10 \\
\hline De 20 - 24 & 26 & 3 & 21 & & 19 & 4 & 26 & & 22 & & 24 & 1 \\
\hline De 25 - 29 & 11 & & 6 & & 5 & 2 & 11 & 1 & 12 & & 6 & 1 \\
\hline De 30 - 34 & 9 & & 5 & & 4 & & 6 & 1 & 5 & & 6 & 1 \\
\hline De 35 - 39 & 1 & & 4 & & 6 & & 4 & & 1 & & 5 & \\
\hline De 40 - 44 & & & 3 & 1 & 2 & & 2 & & & & 3 & \\
\hline De 45 - 49 & & & & & & 2 & & 1 & & 1 & 1 \\
\hline De 50 - 54 & & & & & & & 2 & & & & 2 & \\
\hline De 55-59 & & & & 1 & & 1 & & 2 & 1 & & \\
\hline De 65 - 69 & & & & & & & 1 & & 1 & & & \\
\hline 70 ó Más & 1 & & 3 & & & 1 & & & & & & \\
\hline Total general & 321 & 61 & 348 & 72 & 291 & 48 & 322 & 55 & 345 & 66 & 372 & 66 \\
\hline
\end{tabular}

Fuente. COSED con base en Medicina Legal

Tabla No 8. Delitos sexuales 2008 - 2013 según barrio de ocurrencia

\begin{tabular}{|c|c|c|c|c|c|c|c|}
\hline Barrio & 2008 & 2009 & 2010 & 2011 & 2012 & 2013 & Total general \\
\hline Olaya Herrera & 34 & 39 & 25 & 28 & 29 & 34 & 189 \\
\hline El Pozón & 27 & 21 & 17 & 22 & 26 & 35 & 148 \\
\hline Sin dato & 7 & 18 & 6 & 18 & 10 & 44 & 103 \\
\hline Nelson Mandela & 23 & 20 & 11 & 10 & 22 & 13 & 99 \\
\hline San José de los Campanos & 11 & 9 & 9 & 8 & 14 & 9 & 60 \\
\hline San Fernando & 8 & 11 & 10 & 9 & 8 & 10 & 56 \\
\hline La Boquilla & 7 & 12 & 6 & 7 & 7 & 14 & 53 \\
\hline La María & 9 & 9 & 7 & 8 & 12 & 7 & 52 \\
\hline Zaragocilla & 8 & 13 & 5 & 7 & 5 & 10 & 48 \\
\hline Pasacaballos & 5 & 5 & 8 & 6 & 6 & 10 & 40 \\
\hline Centro & 7 & 8 & 5 & 6 & 7 & 6 & 39 \\
\hline La Candelaria & & 6 & 6 & 8 & 9 & 8 & 37 \\
\hline Bayunca & 5 & 6 & 5 & 11 & 6 & 3 & 36 \\
\hline Daniel Lemaitre & 3 & 4 & 11 & 5 & 4 & 8 & 35 \\
\hline Fredonia & 7 & 5 & 9 & 2 & 6 & 4 & 33 \\
\hline San Pedro Mártir & 10 & 2 & 6 & 10 & 4 & 1 & 33 \\
\hline Boston & 3 & 9 & 2 & 6 & 2 & 8 & 30 \\
\hline Torices & 5 & 4 & 4 & 6 & 7 & 2 & 28 \\
\hline San Francisco & 4 & 3 & 5 & 4 & 6 & 6 & 28 \\
\hline La Esperanza & 5 & 4 & 3 & 5 & 8 & 3 & 28 \\
\hline
\end{tabular}

Fuente. COSED con base en Medicina Legal 
Tabla No 9. Delitos sexuales 2008 - 2013 según tipo de agresor

\begin{tabular}{|c|c|c|c|c|c|c|c|}
\hline Agresor & 2008 & 2009 & 2010 & 2011 & 2012 & 2013 & Total general \\
\hline Agresor dudoso o desconocido & 55 & 116 & 91 & 122 & 128 & 67 & 579 \\
\hline Vecino & 71 & 60 & 59 & 52 & 64 & 69 & 375 \\
\hline Conocido sin ningún trato & 40 & 32 & 31 & 46 & 40 & 59 & 248 \\
\hline $\mathrm{Ns} / \mathrm{Nr}$ - Sin información & 24 & 23 & 14 & 11 & 6 & 101 & 179 \\
\hline Otros familiares civiles o consanguíneos & 19 & 25 & 25 & 17 & 12 & 78 & 176 \\
\hline Padrastro & 32 & 28 & 19 & 22 & 23 & 6 & 130 \\
\hline Amigo (a) & 24 & 28 & 17 & 22 & 15 & 21 & 127 \\
\hline Padre & 16 & 22 & 14 & 12 & 23 & 3 & 90 \\
\hline Novio (a) & 17 & 15 & 7 & 13 & 28 & 1 & 81 \\
\hline Tío (a) & 17 & 17 & 10 & 12 & 16 & 3 & 75 \\
\hline Primo (a) & 12 & 13 & 12 & 13 & 24 & & 74 \\
\hline Abuelo (a) & 8 & 12 & 2 & 3 & 6 & 1 & 32 \\
\hline Profesor (a) & 11 & 3 & 4 & 5 & & 3 & 26 \\
\hline Compañero (a) de trabajo & 1 & 1 & 5 & 11 & 3 & 2 & 23 \\
\hline Hermano (a) & 5 & 3 & 4 & 3 & 4 & 1 & 20 \\
\hline Pareja o Expareja & & & & & & 17 & 17 \\
\hline Cuñado (a) & 4 & 3 & 3 & 1 & 4 & & 15 \\
\hline Ex-novio (a) & 4 & 1 & 4 & 2 & 4 & & 15 \\
\hline Compañero permanente & 1 & 2 & 3 & 3 & 4 & & 13 \\
\hline Compañero de estudio & 3 & 1 & 1 & 1 & 4 & 1 & 11 \\
\hline Arrendatario & & 3 & 2 & 1 & 1 & & 7 \\
\hline Encargado menor & 3 & 1 & & 1 & & 1 & 6 \\
\hline Amante & 2 & 1 & 2 & & 1 & & 6 \\
\hline Ex-amante & 2 & 2 & 2 & & & & 6 \\
\hline Cliente & 1 & 1 & 3 & & & & 5 \\
\hline $\begin{array}{l}\text { Miembros de Fuerzas Armadas, } \\
\text { policía, policía judicial y servicios de } \\
\text { inteligencia }\end{array}$ & & 1 & 1 & 1 & & 2 & 5 \\
\hline Ex esposo (a) & 1 & 2 & & 1 & 1 & & 5 \\
\hline Empleado (a) & 4 & & & 1 & & & 5 \\
\hline Delincuencia común & 1 & 1 & & & & 1 & 3 \\
\hline Proveedor & 1 & 2 & & & & & 3 \\
\hline Pandillas & 1 & & 2 & & & & 3 \\
\hline Arrendador & & & 2 & & & & 2 \\
\hline Hijo (a) & & 1 & & 1 & & & 2 \\
\hline Esposo (a) & 1 & & & & & & 1 \\
\hline Suegro (a) & 1 & & & & & & 1 \\
\hline $\begin{array}{c}\text { Miembro de grupos alzados al margen } \\
\text { de la ley }\end{array}$ & & & & & & 1 & 1 \\
\hline Total general & 382 & 420 & 339 & 377 & 411 & 438 & 2367 \\
\hline
\end{tabular}

Fuente. COSED con base en Medicina Legal

El agresor dudoso y desconocido y el vecino son los agresores más comunes en los delitos sexuales en Cartagena, estos dos tipos de agresores fueron los autores del $40 \%$ del total de casos registrados en el periodo 2008 - 2013, el agresor conocido sin ningún trato y otros familiares civiles y consanguíneos 
Ilustración No 3. Delitos sexuales en Cartagena según barrios de mayor ocurrencia

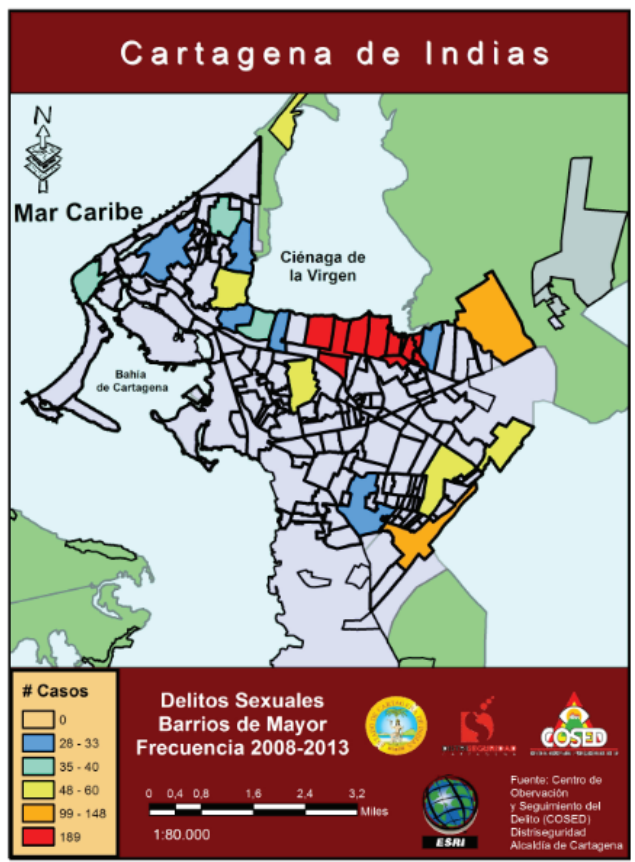

Fuente. Cosed

siguen en orden de mayor frecuencia representando cada uno respectivamente el $10 \mathrm{y}$ 7 por ciento. Aunque hay una participación de agresores ajenos al círculo familiar cercano y que, por el contrario, si hacen parte de la vecindad y los conocidos. Los agresores miembros de la familiar son ampliamente recurrentes, durante este periodo, el $27 \%$ de los casos relacionan agresores que hacen parte del círculo familiar.

\section{VIOLENCIA INTRAFAMILIAR}

En la gráfica 3 se ilustra el comportamiento anual de la Violencia Intrafamiliar en el periodo 2008 - 2013, dividido por cada modalidad: Maltrato a Parejas (MAP), Violencia entre Otros Familiares (VOF) y Maltrato al Menor (MAM). El total de casos denunciados por Violencia Intrafamiliar (VIF) durante el periodo de estudio muestra una tendencia relativamente decreciente, al pasar de 1238 casos en el 2008 a 1092 en el 2013, lo que indica una disminución del $12 \%$; de igual forma, el total de casos ocurridos durante el año 2013 es inferior en un $10 \%$ a los registrados en el 2012. Gráfica No 4. Violencia intrafamiliar
$2008-2013$

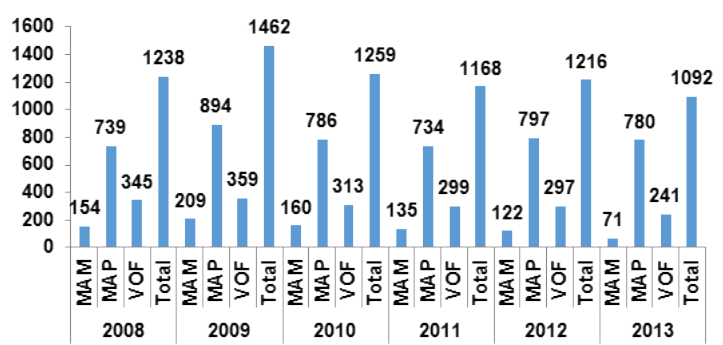

Fuerte. COSED con base en Medicina Legal

Los cifras para cada uno de los años de estudio, señalan que el número total de casos de Maltrato al menor es el más bajo frente a los demás tipos de maltrato (Maltrato de pareja y Violencia entre otros familiares) indica el $11 \%$ de la Violencia intrafamiliar; a su vez, el Maltrato de parejas, representa el mayor número de casos denunciados en los 6 años analizados, constituye el 64\% del total, y es el más común y frecuente con registro en promedio de 788 casos por año.

La Violencia entre otros familiares por su parte, tiene una representatividad en el total de Violencia intrafamiliar del $25 \%$, durante el año 2013 disminuyó el 19\% con respecto al 2012 y el comportamiento en el periodo es decreciente al pasar de 345 casos denunciados en el 2008 a 241 en el 2013.

En forma general, la Violencia Intrafamiliar en el Distrito de Cartagena de Indias es un fenómeno caracterizado por una alta incidencia de casos de Maltrato de Pareja, y es el tipo de violencia familiar que concentra la mayor frecuencia de casos.

También es importante decir, que las denuncias reportadas en el Instituto de Medicina Legal por violencia intrafamiliar son decrecientes o cada vez menores, comprobándose así la baja conciencia y cultura de la denuncia y la existencia de una tolerancia y consentimiento al maltrato dentro de los hogares cartageneros. 
Tabla No 10. Violencia intrafamiliar 2008 - 2013 por edad y sexo de la víctima

\begin{tabular}{|c|c|c|c|c|c|c|c|c|c|c|c|c|}
\hline \multirow{2}{*}{ Edad } & 2008 & & 2009 & & 2010 & & 2011 & & 2012 & & 2013 & \\
\hline & $\mathrm{F}$ & $\mathrm{M}$ & $\mathrm{F}$ & M & $\mathrm{F}$ & $\mathrm{M}$ & $\mathrm{F}$ & M & $\mathrm{F}$ & M & $\mathrm{F}$ & $\mathrm{M}$ \\
\hline De $0-4$ & 14 & 18 & 19 & 21 & 14 & 11 & 13 & 16 & 8 & 9 & 3 & 7 \\
\hline De $5-9$ & 7 & 22 & 19 & 22 & 17 & 29 & 16 & 18 & 10 & 12 & 4 & 11 \\
\hline De $10-14$ & 22 & 24 & 30 & 28 & 29 & 20 & 22 & 20 & 27 & 20 & 24 & 9 \\
\hline De $15-19$ & 96 & 39 & 116 & 39 & 102 & 21 & 89 & 16 & 108 & 20 & 72 & 12 \\
\hline De $20-24$ & 181 & 25 & 192 & 32 & 171 & 30 & 186 & 16 & 174 & 15 & 170 & 11 \\
\hline De $25-29$ & 200 & 26 & 230 & 29 & 183 & 15 & 187 & 26 & 209 & 32 & 195 & 24 \\
\hline De $30-34$ & 170 & 17 & 201 & 35 & 186 & 17 & 156 & 22 & 203 & 21 & 170 & 17 \\
\hline De $35-39$ & 101 & 18 & 133 & 19 & 134 & 21 & 104 & 17 & 98 & 19 & 107 & 18 \\
\hline De $40-44$ & 77 & 26 & 90 & 23 & 77 & 21 & 83 & 8 & 66 & 17 & 77 & 13 \\
\hline De $45-49$ & 51 & 18 & 61 & 24 & 45 & 10 & 47 & 17 & 42 & 17 & 48 & 17 \\
\hline De $50-54$ & 21 & 12 & 24 & 14 & 30 & 13 & 31 & 10 & 34 & 15 & 26 & 4 \\
\hline De $55-59$ & 19 & 9 & 19 & 7 & 20 & 16 & 9 & 11 & 10 & 7 & 9 & 11 \\
\hline De $60-64$ & 8 & 3 & 9 & 8 & 5 & 2 & 5 & 5 & 6 & 6 & 10 & 9 \\
\hline De $65-69$ & 4 & 5 & 3 & 1 & 7 & 3 & 1 & 6 & 2 & 2 & 5 & 2 \\
\hline 70 ó Más & 2 & 3 & 9 & 5 & 6 & 4 & 5 & 6 & 2 & 5 & 1 & 3 \\
\hline Sin dato & & & & & & & & & & & & 3 \\
\hline Total general & 973 & 265 & 1155 & 307 & 1026 & 233 & 954 & 214 & 999 & 217 & 921 & 171 \\
\hline
\end{tabular}

Fuente. COSED con base en Medicina Legal

Las víctimas de Violencia Intrafamiliar en Cartagena, históricamente han sido de sexo femenino, por la alta frecuencia que registra el Maltrato de Parejas, donde en la gran mayoría de los casos las esposas o compañeras permanentes son maltratadas por sus maridos; en los últimos seis años, en el $81 \%$ de los casos ocurridos que fueron denunciados, las víctimas de Violencia Intrafamiliar eran de sexo femenino, contra solo un $19 \%$ de casos en los que las víctimas eran de sexo masculino. En cuanto a las edades, el mayor número de casos se concentra en personas con edades entre los 15 y los 39 años; durante los últimos 6 años, el 71\% de los casos fueron contra personas con estas edades, se identifica así el perfil característico de las víctimas, que corresponde en su mayoría a mujeres jóvenes entre los 15 y 34 años de edad, las cuales son víctimas de maltrato por sus parejas y en una pequeña proporción, jóvenes adolescentes que sufren violencia por parte de sus padres u otros miembros familiares.

Tabla No 11. Violencia intrafamiliar 2008 - 2013 según tipo de agresor

\begin{tabular}{|c|c|c|c|c|c|c|c|}
\hline Agresor & 2008 & 2009 & 2010 & 2011 & 2012 & 2013 & Total general \\
\hline Compañero permanente & 704 & 853 & 676 & 586 & 637 & 310 & 3766 \\
\hline Esposo (a) & 108 & 101 & 124 & 100 & 86 & 42 & 561 \\
\hline Hermano (a) & 98 & 114 & 70 & 63 & 68 & 27 & 440 \\
\hline Ex - Compañero (a) & & 1 & & & & 370 & 371 \\
\hline Padre & 73 & 77 & 63 & 64 & 49 & 22 & 348 \\
\hline Cuñado (a) & 59 & 55 & 54 & 66 & 49 & 10 & 293 \\
\hline Hijo (a) & 45 & 45 & 47 & 34 & 27 & 17 & 215 \\
\hline Madre & 29 & 49 & 35 & 29 & 29 & 7 & 178 \\
\hline Ex esposo (a) & & 1 & 38 & 34 & 87 & 18 & 178 \\
\hline Tío (a) & 25 & 36 & 36 & 35 & 24 & 8 & 164 \\
\hline Novio (a) & 22 & 28 & 32 & 33 & 22 & 15 & 152 \\
\hline Primo (a) & 21 & 27 & 29 & 22 & 31 & 13 & 143 \\
\hline Ex-novio (a) & & & 2 & 57 & 58 & 18 & 135 \\
\hline Sin dato & & 1 & & & & 133 & 134 \\
\hline Padrastro & 22 & 19 & 17 & 19 & 19 & 8 & 104 \\
\hline
\end{tabular}


DIAGNÓSTICO GENERAL DE LA SITUACIÓN DE VIOLENCIA Y CRIMINALIDAD EN CARTAGENA DE INDIAS. 2008 - 2013

\begin{tabular}{|c|c|c|c|c|c|c|c|}
\hline Amante & 20 & 37 & 22 & 5 & 5 & 3 & 92 \\
\hline Suegro (a) & 8 & 5 & 2 & 7 & 8 & 6 & 36 \\
\hline Familiar & & & & & & 31 & 31 \\
\hline Madrastra & 2 & 8 & 4 & 1 & 8 & & 23 \\
\hline Ex - Novio (a) & & & & & & 19 & 19 \\
\hline Ex-amante & & & 1 & 9 & 6 & & 16 \\
\hline Abuelo (a) & 1 & 1 & 5 & 2 & 3 & & 12 \\
\hline otros & & 4 & 2 & 2 & & & 8 \\
\hline Yerno & & & & & & 4 & 4 \\
\hline Encargado del menor & 1 & & & & & 3 & 4 \\
\hline Nieto & & & & & & 2 & 2 \\
\hline Conocido sin ningún trato & & & & & & 2 & 2 \\
\hline Sobrino (a) & & & & & & 2 & 2 \\
\hline Vecino & & & & & & 1 & 1 \\
\hline Custodio & & & & & & 1 & 1 \\
\hline Total general & 1238 & 1462 & 1259 & 1168 & 1216 & 1092 & 7435 \\
\hline
\end{tabular}

Fuente. COSED con base en Medicina Legal

En relación con el agresor, se observa que el compañero permanente y el esposo tienden a propinar con mayor frecuencia el maltrato por el que se denunció la Violencia Intrafamiliar en la ciudad; mientras el compañero permanente generó cerca del $51 \%$ de los casos, el esposo lo hizo en un $8 \%$, pero también tienen participación en el maltrato provocado a las víctimas, el hermano con el $6 \%$ del total, el excompañero, con 5\%, el padre el 5\% y el cuñado, con el 4\%, entre los de mayor representación.

La distribución de los agresores de la VIF presentados durante el año 2012, resulta ser la mismas desde el año 2008, como lo muestra la tabla 7, el orden de mayor frecuencia es el mismo, prevaleciendo el compañero permanente como el principal victimario, quien agrede a más de la mitad de las víctimas que son reportadas.

Tabla No 12. Violencia intrafamiliar 2008 - 2013 según razón de la violencia

\begin{tabular}{|c|c|c|c|c|c|c|c|}
\hline Razón de la violencia & 2008 & 2009 & 2010 & 2011 & 2012 & 2013 & Total general \\
\hline Intolerancia & 551 & 648 & 521 & 496 & 604 & 349 & 3169 \\
\hline Celos & 143 & 163 & 195 & 169 & 157 & 276 & 1103 \\
\hline Sin dato & 93 & 76 & 103 & 154 & 2 & 343 & 771 \\
\hline Alcoholismo & 111 & 142 & 84 & 69 & 79 & 60 & 545 \\
\hline Desamor & 126 & 156 & 108 & 75 & 41 & 1 & 507 \\
\hline NS/NR - Sin información & 33 & 36 & 42 & 46 & 169 & 21 & 347 \\
\hline Machismo & 51 & 63 & 64 & 47 & 52 & 13 & 290 \\
\hline Consumo de sustancias psicoactivas & 56 & 60 & 48 & 35 & 32 & 9 & 240 \\
\hline Económicas & 32 & 52 & 37 & 39 & 26 & 2 & 188 \\
\hline Otras razones & 23 & 27 & 29 & 17 & 27 & & 123 \\
\hline Infidelidad & 5 & 16 & 17 & 17 & 25 & 4 & 84 \\
\hline Desconfianza & 12 & 21 & 11 & 2 & 2 & & 48 \\
\hline $\begin{array}{c}\text { Alcohol consumo de sustancias } \\
\text { piscoactivas }\end{array}$ & & & & & 9 & 9 \\
\hline Abandono e intolerancia & & & & & & 4 & 4 \\
\hline Enfermedad física o mental & 2 & 2 & & & & & 4 \\
\hline Separación / Divorcio & & & & 2 & & & 2 \\
\hline PROBLEMAS FAMILIARES & & & & & & 1 & 1 \\
\hline Total general & 1238 & 1462 & 1259 & 1168 & 1216 & 1092 & 7435 \\
\hline
\end{tabular}

Fuente. COSED con base en Medicina Legal 
Ilustración No 4. Violencia Intrafamiliar en Cartagena según barrios de mayor ocurrencia

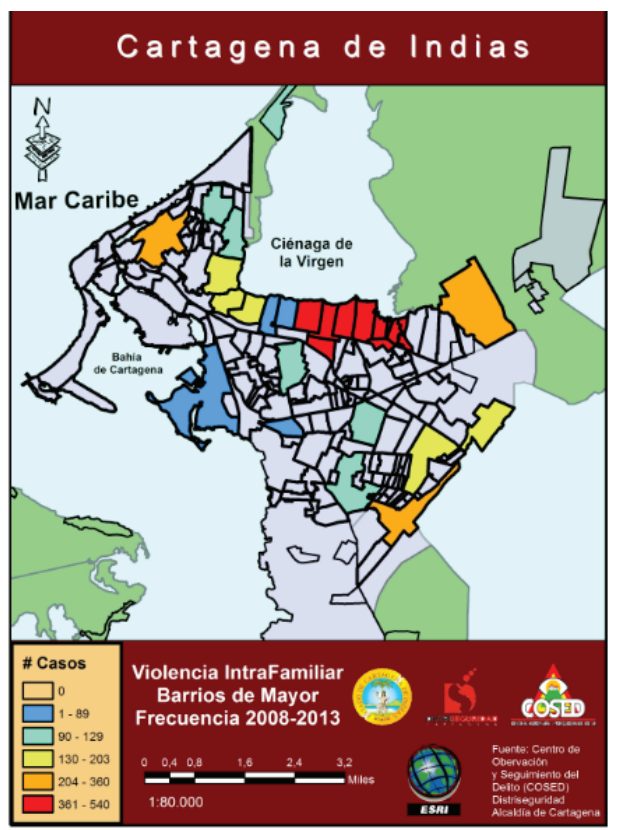

Fuente. Cosed
La principal razón causante de la violencia intrafamiliar es la intolerancia, que durante el periodo 2008 - 2013 reportó 3169 casos de los 7435 totales, siendo entonces la intolerancia la razón del $43 \%$ de los casos de Violencia Intrafamiliar que se registran dentro de los hogares cartageneros y fueron reportados en Medicina Legal, en su orden le sigue los celos, que fueron la razón del 15\%, y luego el alcoholismo, por lo que fueron maltratadas el $7 \%$ de las víctimas.

Estos factores que se han considerado razones de la violencia y que en general se relacionan, por una parte, con la incapacidad de los miembros masculinos del hogar para tolerar situaciones recurrentes y resultantes de la convivencia con sus familiares; y por otra, con el consumo del alcohol. Son la aproximación más cercana a las posibles causas del fenómeno y hacen parte de un grupo de acciones recurrentes, costumbres y mal manejo de las emociones humanas con las que los individuos a diario confrontan las dificultades en la convivencia y la relaciones al interior del hogar.

Tabla No 13. Violencia intrafamiliar 2008 - 2013 según barrio de ocurrencia

\begin{tabular}{|c|c|c|c|c|c|c|c|}
\hline Barrio & 2008 & 2009 & 2010 & 2011 & 2012 & 2013 & Total general \\
\hline Olaya Herrera & 80 & 111 & 78 & 84 & 96 & 91 & 540 \\
\hline El Pozón & 48 & 73 & 71 & 47 & 56 & 65 & 360 \\
\hline Torices & 53 & 40 & 39 & 30 & 48 & 45 & 255 \\
\hline Nelson Mandela & 48 & 35 & 40 & 36 & 35 & 38 & 232 \\
\hline San Fernando & 42 & 33 & 32 & 44 & 32 & 20 & 203 \\
\hline La María & 28 & 32 & 28 & 31 & 19 & 22 & 160 \\
\hline La Candelaria & 31 & 28 & 24 & 25 & 23 & 28 & 159 \\
\hline La Esperanza & 29 & 28 & 28 & 22 & 15 & 28 & 150 \\
\hline San José de los Campanos & 26 & 29 & 21 & 23 & 33 & 16 & 148 \\
\hline San Pedro Mártir & 26 & 16 & 16 & 26 & 22 & 23 & 129 \\
\hline Zaragocilla & 26 & 30 & 17 & 19 & 15 & 19 & 126 \\
\hline Daniel Lemaitre & 18 & 30 & 27 & 12 & 20 & 18 & 125 \\
\hline Pasacaballos & 20 & 38 & 13 & 15 & 20 & 17 & 123 \\
\hline La Boquilla & 12 & 17 & 19 & 22 & 20 & 29 & 119 \\
\hline San Francisco & 26 & 13 & 26 & 18 & 17 & 18 & 118 \\
\hline Blas de Lezo & 17 & 28 & 21 & 20 & 14 & 17 & 117 \\
\hline Boston & 4 & 24 & 16 & 14 & 21 & 10 & 89 \\
\hline Republica del Líbano & 19 & 15 & 15 & 15 & 9 & 16 & 89 \\
\hline Bosque & 14 & 22 & 26 & 15 & 12 & & 89 \\
\hline Ceballos & 13 & 28 & 12 & 10 & 12 & 11 & 86 \\
\hline
\end{tabular}

Fuente. COSED con base en Medicina Legal 
En cuanto a la distribución territorial, la tabla 9 muestra los 20 barrios con mayor frecuencia de casos en la ciudad, de los cuales se destacan 5 que sobrepasan los 200 casos en el periodo analizado, y de un promedio anual por encima de los 40 registros, entre ellos, el barrio Olaya Herrera, el de mayor cantidad, con 540, el 7\% de los ocurridos y denunciados y aproximadamente 90 de promedio anual, le siguen en su orden El Pozón, con un registro menor que alcanza los 360 y un promedio anual de 65 denuncias, Torices con 45 casos promedio en el año y Nelson Mandela y San Fernando con una cifra levemente menor.

Los barrios relacionados en la Violencia Intrafamiliar en promedio pertenecen al estrato 1 y 2 y son también el escenario de eventos violentos como los homicidios, la violencia interpersonal y los delitos sexuales. Se concentran en gran medida en las comunas 4,5 y 6 y tienen la particularidad de tener alta población. Los barrios Olaya Herrera, El Pozón, Nelson Mandela y Torices, son 4 de los más grandes barrios de la ciudad.

\section{VIOLENCIA INTERPERSONAL}

La Violencia Interpersonal corresponde a aquellas riñas registradas entre personas o grupos de

\section{Gráfica No 5. Violencia interpersonal $2008-2013$}

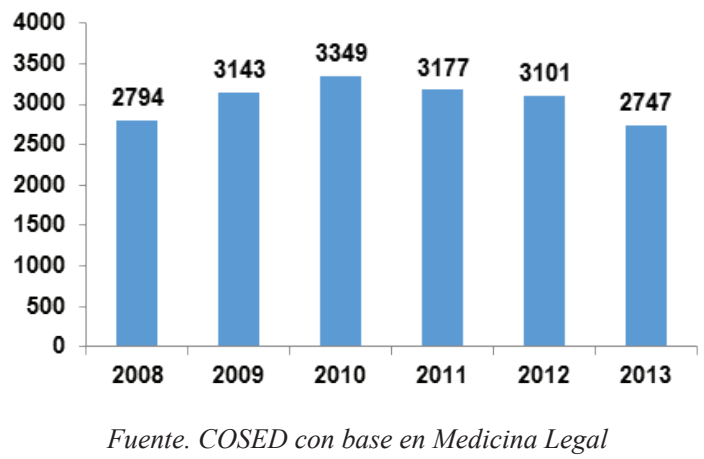

personas donde se presenta algún tipo de agresión física y que en esta clasificación no ocurren dentro del hogar o entre miembros del mismo.

En Cartagena en el periodo 2008 - 2013 fueron reportadas al Instituto de Medicina Legal 18.311 víctimas de Violencia Interpersonal, los casos como se observa en la gráfica 4 vienen registrando tendencia decreciente desde el año 2010, cuando se tuvo el mayor registro del periodo, 3349 casos en total, hasta llegar al menor registro de los 6 años analizados, 2747 casos en el año 2013. En promedio al año son reportados 3051 casos y solo en dos años en la serie analizada la cifra estuvo por debajo de dicho promedio, años 2008 y 2013.

Tabla No 14. Violencia interpersonal 2008 - 2013 por edad y sexo de la víctima

\begin{tabular}{|c|c|c|c|c|c|c|c|c|c|c|c|c|}
\hline \multirow{2}{*}{ Edad } & \multicolumn{2}{|c|}{2008} & \multicolumn{2}{|c|}{2009} & \multicolumn{2}{|c|}{2010} & \multicolumn{2}{|c|}{2011} & \multicolumn{2}{|c|}{2012} & \multicolumn{2}{|c|}{2013} \\
\hline & $\mathrm{F}$ & $\mathrm{M}$ & $\mathrm{F}$ & $\mathrm{M}$ & $\mathrm{F}$ & M & $\mathrm{F}$ & $\mathrm{M}$ & $\mathrm{F}$ & $\mathrm{M}$ & $\mathrm{F}$ & $\mathrm{M}$ \\
\hline De $0-4$ & 5 & 6 & 4 & 11 & 5 & 9 & 9 & 10 & 7 & 13 & 7 & 6 \\
\hline De 5-9 & 3 & 5 & 9 & 19 & 4 & 8 & 6 & 20 & 6 & 12 & 9 & 17 \\
\hline De $10-14$ & 30 & 28 & 17 & 44 & 38 & 62 & 37 & 56 & 44 & 52 & 50 & 48 \\
\hline De $15-19$ & 119 & 234 & 153 & 261 & 181 & 321 & 157 & 313 & 168 & 275 & 134 & 223 \\
\hline De $20-24$ & 232 & 376 & 251 & 407 & 250 & 385 & 244 & 387 & 243 & 368 & 172 & 316 \\
\hline De 25 - 29 & 226 & 290 & 236 & 305 & 217 & 334 & 228 & 317 & 213 & 309 & 192 & 295 \\
\hline De 30 - 34 & 157 & 177 & 190 & 231 & 215 & 271 & 188 & 262 & 175 & 242 & 127 & 217 \\
\hline De 35 - 39 & 125 & 164 & 143 & 160 & 147 & 197 & 126 & 153 & 136 & 143 & 112 & 172 \\
\hline De $40-44$ & 92 & 136 & 108 & 153 & 107 & 139 & 91 & 139 & 92 & 133 & 94 & 132 \\
\hline De $45-49$ & 57 & 91 & 83 & 101 & 60 & 106 & 68 & 91 & 67 & 112 & 66 & 80 \\
\hline De $50-54$ & 39 & 75 & 35 & 73 & 52 & 86 & 51 & 80 & 52 & 74 & 35 & 76 \\
\hline De $55-59$ & 19 & 37 & 23 & 51 & 23 & 46 & 23 & 45 & 31 & 38 & 26 & 48 \\
\hline De $60-64$ & 6 & 29 & 10 & 23 & 8 & 26 & 8 & 36 & 15 & 34 & 13 & 33 \\
\hline De $65-69$ & 3 & 17 & 11 & 11 & 3 & 17 & 7 & 7 & 10 & 12 & 9 & 20 \\
\hline 70 ó Más & 6 & 9 & 5 & 8 & 13 & 16 & 8 & 10 & 12 & 13 & 6 & 12 \\
\hline Sin dato & & 1 & 3 & 4 & 2 & 1 & & & & & & \\
\hline Total general & 1119 & 1675 & 1281 & 1862 & 1325 & 2024 & 1251 & 1926 & 1271 & 1830 & 1052 & 1695 \\
\hline
\end{tabular}


El perfil de las víctimas de Violencia Interpersonal son mayoritariamente hombres con edades entre los 15 y 49 años, de manera que las riñas en la ciudad de Cartagena se presentan entre personas relativamente jóvenes y existe una importante participación de menores de edad en este tipo de conflictos. Las edades que particularmente concentran el mayor número de víctimas corresponden al rango etario de los 20 a los 24 años, que tuvo el $20 \%$ del total, seguido por el rango de los 25 a los 29 años donde hubo el 17\% del total de las víctimas. Víctimas de sexo femenino fueron el $40 \%$ frente al restante $60 \%$ que correspondió a sexo opuesto, es decir, que aunque existe una incidencia de casos de sexo masculino que supera la mitad de los registros, la incidencia de casos de sexo femenino tiene también una importante participación.

Tabla No 15. Violencia interpersonal 2008 - 2013 según barrio de ocurrencia

\begin{tabular}{|c|c|c|c|c|c|c|c|}
\hline Barrio & 2008 & 2009 & 2010 & 2011 & 2012 & 2013 & Total general \\
\hline Olaya Herrera & 228 & 258 & 259 & 202 & 191 & 229 & 1367 \\
\hline El Pozón & 124 & 144 & 145 & 111 & 131 & 121 & 776 \\
\hline Centro & 81 & 92 & 115 & 109 & 94 & 65 & 556 \\
\hline Nelson Mandela & 65 & 66 & 85 & 65 & 54 & 75 & 410 \\
\hline La Esperanza & 61 & 50 & 80 & 73 & 72 & 58 & 394 \\
\hline Bosque & 85 & 71 & 74 & 61 & 56 & 36 & 383 \\
\hline Pasacaballos & 58 & 72 & 72 & 44 & 63 & 56 & 365 \\
\hline La Candelaria & 33 & 57 & 70 & 87 & 59 & 57 & 363 \\
\hline San francisco & 58 & 44 & 66 & 75 & 53 & 55 & 351 \\
\hline La Boquilla & 53 & 47 & 57 & 63 & 67 & 53 & 340 \\
\hline Torices & 35 & 50 & 58 & 45 & 60 & 88 & 336 \\
\hline La María & 50 & 64 & 54 & 54 & 63 & 39 & 324 \\
\hline San Fernando & 51 & 73 & 54 & 65 & 39 & 37 & 319 \\
\hline Bocagrande & 44 & 36 & 66 & 34 & 54 & 61 & 295 \\
\hline San José de los Campanos & 45 & 42 & 44 & 49 & 53 & 58 & 291 \\
\hline Zaragocilla & 51 & 46 & 51 & 42 & 55 & 46 & 291 \\
\hline Daniel Lemaitre & 40 & 64 & 49 & 47 & 46 & 42 & 288 \\
\hline Boston & 46 & 38 & 46 & 53 & 36 & 44 & 263 \\
\hline Sin dato & 53 & 60 & 80 & 31 & 23 & 15 & 262 \\
\hline Blas de Lezo & 38 & 38 & 47 & 44 & 33 & 38 & 238 \\
\hline
\end{tabular}

Fuente. COSED con base en Medicina Legal

Olaya Herrera y El Pozón son también los barrios de mayor frecuencia de víctimas en Violencia Interpersonal, como lo es en los homicidios y la Violencia intrafamiliar. De igual forma, también aparece Nelson Mandela y barrios como la Esperanza, El Bosque, La Candelaria, Torices y San Fernando, entre otros, en la lista de los 20 barrios de la ciudad con mayor frecuencia, en el casos particular de este tipo de violencia. Se destaca la presencia del barrio Centro, que en promedio es de estrato 3 y 4 a diferencias de la mayoría que corresponden a los estratos 1 y 2 . El Centro es el tercer barrio de la ciudad con mayor número de casos denunciados por Violentica Interpersonal, durante el periodo 2008 - 2013 registró 556 casos, 800 menos que
Olaya Herrera y 220 menos que El Pozón y, por lo contrario, presento 146 más que Nelson Mandela y 150 más que La Esperanza, barrios que en los otros tipos de violencia suelen tener mayor participación.

\section{DELITOS CONTRA LA PROPIEDAD Y EL PATRIMONIO}

\section{HURTOS}

Durante el año 2013 en la ciudad de Cartagena hubo un total de hurtos de 1815 , de los cuales el $62 \%$ fueron hurtos a personas, el $16 \%$ hurtos a residencias, $12 \%$ a comercio, el $9 \%$ a motocicletas y el $1 \%$ a vehículos. En todo 
Ilustración No 5. Violencia Intrafamiliar en Cartagena según barrios de mayor ocurrencia

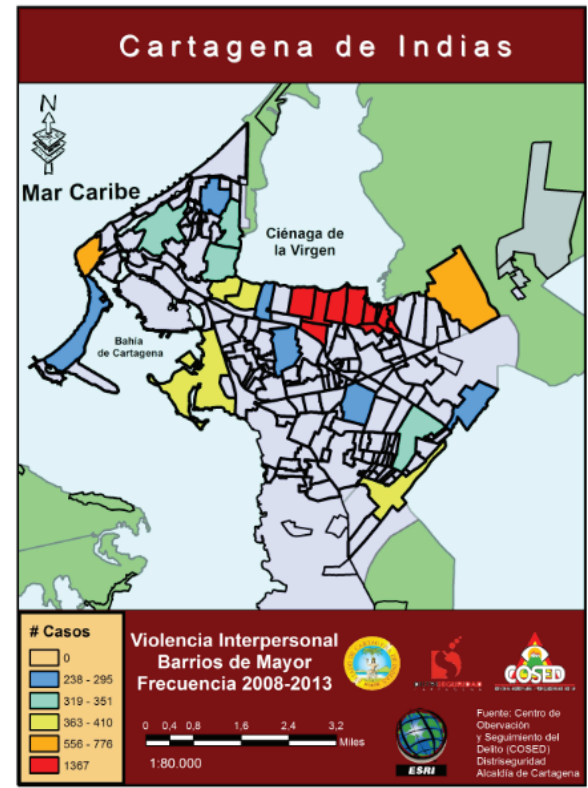

Fuente. Cosed

Gráfica No 6. Hurtos 2008 - 2013

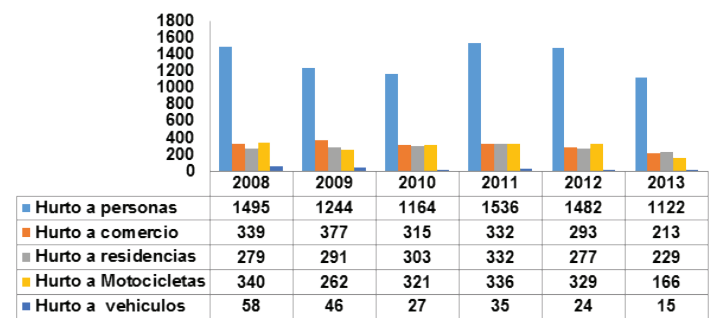

Fuente. COSED con base en Policía Nacional

el periodo analizado, los hurtos a personas representan la mayoría del total de hurtos ocurridos y denunciados en la ciudad, su participación en el total general es del 59\%. La tendencia de los hurtos denunciados, en forma general, durante los 6 años analizados es relativamente decreciente al pasar de 2511 casos en el 2008 a 1815 en el 2013, indicando una disminución del 28\%. Este comportamiento decreciente en los casos denunciados también se identifica en cada uno de los tipos de hurtos, con una notable diferencia en los hurtos a personas registrados en el 2011 y 2013, cuando las cifras fueron levemente superiores a las reportadas en los demás años.

\section{Ilustración No 6. Hurtos por barrios principales modalidades}
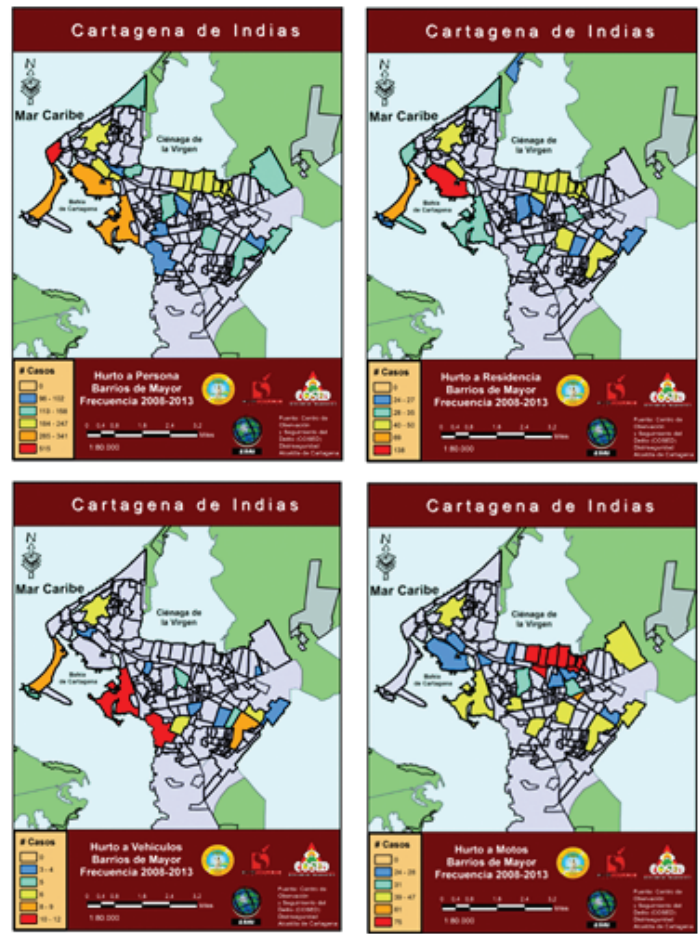

Fuente. Cosed

La distribución de los hurtos por barrio permite constatar que la frecuencia de algunos de estos hurtos tiene algún tipo de relación con los barrios en los que suceden; por ejemplo, es común que el hurto a personas sea recurrente en cualquier barrio de la ciudad, pero que se hagan muy presentes en barrios como Manga y Bocagrande y que tengan una frecuencia menor en los barrios ubicados en la comuna 4, 5 y 6 . Mientras que el hurto a residencias y a vehículos es más frecuente en barrios como Manga y Bosque, mientras que el hurto a motocicletas es común en los barrios pertenecientes a la Comuna 4, 5 y 6, es decir, Olaya Herrera, La Esperanza y La María.

\section{EXTORSIONES}

El delito de extorsión en Cartagena a través de los años ha venido aumentando su frecuencia, llegar hasta convertirse en una forma delictiva de alto impacto en la seguridad de los ciudadanos, aunque aún los registro de denuncias son muy bajos, la tendencia en los últimos años ha sido 
Gráfica No 7. Extorsiones 2008 - 2013

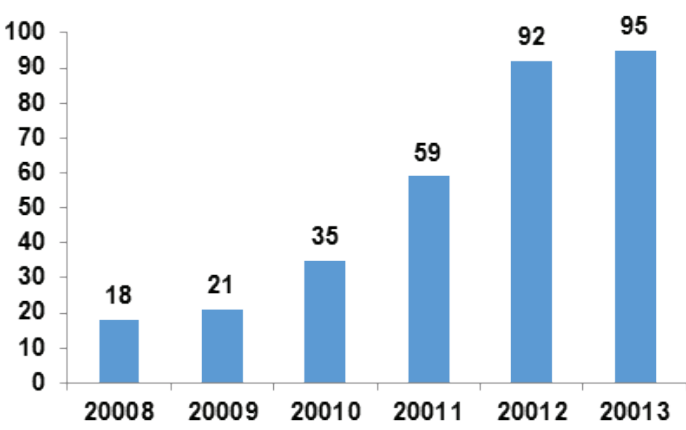

creciente, y ha llegado a multiplicarse. Como lo muestra la gráfica 7, mientras en el año 2008 solo se registraron 18 denuncias por extorsión en la ciudad, durante el año 2013 fueron 95, esto indica un incremento exponencial y el comportamiento año tras años es ampliamente decreciente; sin embargo, aún existe miedo en la ciudadanía por hacer uso del derecho a denuncia y darle participación a las autoridades para el manejo e investigación de este tipo de delito.

Fuente. COSED con base en GAULA de la Policía

Tabla No 16. Extorsiones 2008 - 2013 por modalidad

\begin{tabular}{|c|c|c|c|c|c|c|c|}
\hline Modalidad & $\mathbf{2 0 0 8}$ & $\mathbf{2 0 0 9}$ & $\mathbf{2 0 1 0}$ & $\mathbf{2 0 1 1}$ & $\mathbf{2 0 1 2}$ & $\mathbf{2 0 1 3}$ & Total general \\
\hline Clásica & 18 & 19 & 25 & 29 & 35 & 59 & 185 \\
\hline Micro extorsión & & & 3 & 26 & 45 & 25 & 99 \\
\hline Carcelaria & & 1 & 4 & 1 & 8 & 9 & 23 \\
\hline Información íntima & & 1 & 3 & 2 & 2 & & 8 \\
\hline Devolución documentos & & & & & 1 & & 1 \\
\hline Brindar protección & & & & 1 & & & 1 \\
\hline Devolución de bienes & & & & & 1 & 2 & 3 \\
\hline Total general & $\mathbf{1 8}$ & $\mathbf{2 1}$ & $\mathbf{3 5}$ & $\mathbf{5 9}$ & $\mathbf{9 2}$ & $\mathbf{9 5}$ & $\mathbf{3 2 0}$ \\
\hline
\end{tabular}

Fuente. COSED con base en GAULA de la Policia

La modalidad más común en las extorsiones es la clásica, durante el periodo de estudio representó el $58 \%$ del total de casos, seguido por la micro extorsión que representó 31\% y la modalidad carcelaria que tiene una participación del 7\%.

Tabla No 17. Extorsiones 2008 - 2013 por modus operandi

\begin{tabular}{|c|c|c|c|c|c|c|c|}
\hline Modus operandi & $\mathbf{2 0 0 8}$ & $\mathbf{2 0 0 9}$ & $\mathbf{2 0 1 0}$ & $\mathbf{2 0 1 1}$ & $\mathbf{2 0 1 2}$ & $\mathbf{2 0 1 3}$ & Total general \\
\hline Llamadas a celular & 18 & 6 & 17 & 22 & 27 & 33 & 123 \\
\hline Directa & & 7 & 4 & 21 & 33 & 36 & 101 \\
\hline Mixta & & 1 & 5 & 14 & 27 & 23 & 70 \\
\hline Llamadas a fijo & & 4 & 2 & 1 & 3 & 1 & 11 \\
\hline Cartas & & 2 & 3 & & 1 & 1 & 7 \\
\hline Mensajes a email & & & 4 & 1 & & & 5 \\
\hline Mensajes de texto & & & 1 & & 1 & 1 & 3 \\
\hline Total general & $\mathbf{1 8}$ & $\mathbf{2 0}$ & $\mathbf{3 6}$ & $\mathbf{5 9}$ & $\mathbf{9 2}$ & $\mathbf{9 5}$ & $\mathbf{3 2 0}$ \\
\hline
\end{tabular}

Fuente. COSED con base en GAULA de la Policía

En cuanto al modus operandi, las llamadas a celular es la forma más común en que operan los extorsionistas, durante el periodo 2008 - 2013, 123 casos de los denunciados, correspondientes 
DIAGNÓSTICO GENERAL DE LA SITUACIÓN DE VIOLENCIA Y CRIMINALIDAD EN CARTAGENA DE INDIAS. 2008 - 2013

al 38\% fueron de esta manera, seguido por la extorsión directa que reportó 101 casos, el 32\% del total y la modalidad mixta que representó el
$22 \%$; el resto se distribuye en cuatro distintas modalidades que se relacionan en la tabla 32 .

\section{REFERENCIAS BIBLIOGRÁFICAS}

Becker, G. (Marzo de 1968). El Crimen y el Castigo: Un Enfoque Económico. Recuperado el 22 de Marzo de 2013, de National Bureau of Economic Research: http://www.nber.org/chapters/c3625.pdf

Goyeneche, F., Pardo, J., \& Mármol, O. (2011). Valoración Económica del delito de homicidio en Cartagena. . Cartagena de Indias: Revista Panorama Económico. No. 18.

Hurtado, J. (2010). Metodología de la investigación. Guía para la comprension holística de la ciencia. Bogotá - Caracas: Quirón Editores.

Rubio, M. (Noviembre de 1997). Costos de la violencia en Colombia. Recuperado el 5 de Febrero de 2013, de Universidad de Los Andes:http://economia.uniandes.edu.co/content/download/9313/45810/file/costos_violencia.pdf. 